\title{
Modeling of the Deformation Dynamics of Single and Twin Fluid Droplets Exposed to Aerodynamic Loads
}

\author{
L. Wieth*, S. Braun, G. Chaussonnet, T. F. Dauch, M. Keller, C. Höfler, R. Koch, H.-J. Bauer \\ Institut für Thermische Strömungsmaschinen (ITS), \\ Karlsruher Institut für Technologie (KIT), Karlsruhe, Germany \\ ${ }^{*}$ Corresponding author: lars.wieth@kit.edu
}

\begin{abstract}
Droplet deformation and breakup plays a significant role in liquid fuel atomization processes. The droplet behavior needs to be understood in detail, in order to derive simplified models for predicting the different processes in combustion chambers. Therefore, the behavior of single droplets at low aerodynamic loads was investigated using the Lagrangian, mesh-free Smoothed Particle Hydrodynamics (SPH) method. The simulations to be presented in this paper are focused on the deformation dynamics of pure liquid droplets and fuel droplets with water added to the inside of the droplet. The simulations have been run at two different relative velocities.

As SPH is relatively new to Computational Fluid Dynamics (CFD), the pure liquid droplet simulations are used to verify the SPH code by empirical correlations available in literature. Furthermore, an enhanced characteristic deformation time is proposed, leading to a good description of the temporal initial deformation behavior for all investigated test cases. In the further course, the deformation behavior of two fluid droplets are compared to the corresponding single fluid droplet simulations. The results show an influence of the added water on the deformation history. However, it is found that, the droplet behavior can be characterized by the pure fuel Weber number.
\end{abstract}

\section{Introduction}

For an optimization of modern gas turbines, the atomization process needs to be understood in detail. The present investigation focuses on the behavior of single droplets at low aerodynamic loads. These conditions occur right after the jet breakup in a jet-in-crossflow configuration for example. Hence, they are crucial for the following evaporation and combustion process. Various experimental investigations of the behavior of droplets at aerodynamic loads have been conducted in the past, e.g. [1, 2, 3]. However, the experimental setup, which either relays on a shock tube experiment or a free falling droplet in a crossflow, does not allow for a detailed insight of the phenomena involved in the deformation and breakup process. Therefore, numerical investigations have been conducted to gain insight into the underlying physics, e.g. [4, 5, 6].

In order to predict all processes occurring in combustion chambers, from the liquid fuel injection to the combustion, commonly Euler-Lagrange methods are used. These methods predict the air flow on an Eulerian mesh, while the liquid fuel is inserted as Lagrangian parcels. To describe the behavior of the liquid fuel droplets, simplified models were derived using experimental and detailed numerical investigations. The most common models to describe the initial deformation phase are the Normal-Mode (NM) model and the Non-linear Taylor Analogy Breakup (NLTAB) model [7] 8], which is a nonlinear extension to the well known TAB model proposed by O'Rouke [9]. In all models it is assumed that after reaching a critical deformation, the Lagrangian parcel will undergo secondary breakup, which is described by empirical models as well (e.g. [10]).

The assumption of such empirical models is the droplet is exposed to a quasi-steady aerodynamic load. Therefore, the history and the temporal evolution of the droplet deformation is considered. This may lead to unphysical droplet drag predictions. In the present paper the temporal evolution of droplet deformation is investigated at low aerodynamic loads using the Lagrangian, mesh-free Smoothed Particle Hydrodynamics (SPH) method. The weakly compressible SPH code in use was developed and validated in order to predict the atomization process in gas turbine engines [11]. The main advantage of SPH over mesh-based methods is the inherent interface advection without the need of an interface capturing algorithm. Furthermore, the effect of water added to the inside of the liquid fuel droplet is investigated. Preliminary tests performed in heavy duty gas turbines showed that the addition of water has a positive effect on the thermal NOx emissions[12. The addition of water to the fuel oil not only decreases the combustion temperature due to the heat of evaporation, but has a positive effect on the atomization process as well [13].

Therefore, the deformation of single, emulsified fuel droplets with an initial diameter of $d_{0} \approx 60 \mu \mathrm{m}$ with different water volume fractions $\phi=V_{\mathrm{w}} /\left(V_{\mathrm{w}}+V_{\text {Oil }}\right)(\phi=0, \phi=0.23, \phi=1)$ exposed to different air velocities $\left(\left|\vec{v}_{\text {Air }}\right|=22.5 \mathrm{~m} / \mathrm{s}\right.$ and $\left|\vec{v}_{\text {Air }}\right|=24.34 \mathrm{~m} / \mathrm{s}$ ) are investigated. Furthermore, the placement of the water inside the droplet is varied to determine its influence on the droplet deformation.

\section{Methodology}

The full Lagrangian, mesh-free Smoothed Particle Hydrodynamics (SPH) method has been developed in the late 1970s for the simulation of non-axisymmetric phenomena in astrophysics [14 15]. 
In this approach integral equations or partial differential equations with boundary conditions are solved over an arbitrarily scattered set of movable discretization points. Those so called particles represent a finite volume of the fluid domain on a continuum scale. The physical quantities, e.g. position and velocity, as well as the fluid properties are assigned to the particles. The interaction of the particles is taken into account by a weighting function. Therefore, in contrast to common grid based techniques no complicated spatial discretization schemes are required. This is advantageous for the simulation of problems with free surfaces, high deformations and moving surfaces.

In the following the basics of the SPH method, which are needed to solve the conservation equations, will be presented.

\section{SPH Formulation}

The fact, that every spatial function $f(\vec{x})$ can be exactly reproduced by the convolution of the function itself with the Dirac delta function $\delta\left(\vec{x}-\vec{x}^{\prime}\right)$ is the basis of the SPH-interpolation:

$$
f(\vec{x})=\int_{V} f\left(\vec{x}^{\prime}\right) \delta\left(\vec{x}-\vec{x}^{\prime}\right) d x^{\prime} .
$$

The determination of a quantity at position $\vec{x}$ requires the quantities at the surrounding positions $\vec{x}^{\prime}$ to be taken into account. Since the Dirac function is only valid at one point, it is replaced by a smooth weight function with similar properties, the so called kernel $W\left(\vec{x}-\vec{x}^{\prime}, h\right)$. The kernel assigns a weight to the neighboring particles depending on their distance $\left(\vec{x}-\vec{x}^{\prime}\right)$ from the center particle. To ensure stability and consistency of the method, the kernel has to fulfill certain requirements [16]. The kernel is compact. The maximum radius of influence is defined by the smoothing length $h$. In Figure 1 the interpolation of a function at the position of particle i through the known functions at the positions of neighboring particles $\mathrm{j}$ is illustrated.

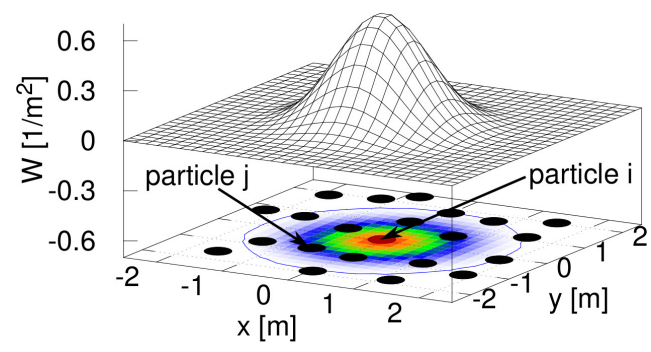

Figure 1. Interpolation for the center particle (red) with a kernel function $\mathrm{W}$

For the numerical determination of a quantity the integral approximation is replace by the summation over all neighbor particles j, the so called quadrature [17]:

$$
f(\vec{x})=\sum_{j} f\left(\vec{x}_{j}\right) V_{j} W\left(\vec{x}_{i}-\vec{x}_{j}, h\right)=\sum_{j} f\left(\vec{x}_{j}\right) \frac{m_{j}}{\rho_{j}} W\left(\vec{x}_{i}-\vec{x}_{j}, h\right),
$$

where $V$ is the volume, $m$ is the mass and $\rho$ the density of the particles.

When using a differentiable kernel, the partial derivative of a function $\vec{\nabla} f(\vec{x})$ is given by:

$$
\vec{\nabla} f(\vec{x})=\sum_{j} f\left(\vec{x}_{j}\right) \frac{m_{j}}{\rho_{j}} \vec{\nabla} W\left(\vec{x}_{i}-\vec{x}_{j}, h\right)
$$

This is only valid if the domain of interpolation of a particle is not truncated by the boundary of the computational domain.

\section{SPH formulation of the Navier-Stokes equations}

The mathematical characterization of macroscopic flows are described by the Navier-Stokes-equations. They include thee conservation equations: the continuity, momentum and energy equation, whereof the later will be neglected, because the flow is considered as isothermal.

Different SPH formulations of the Navier-Stokes equations can be found in literature based on the SPH-formulations (2) and 3 and further mathematical transformations [17]. The approximation of the density and the pressure gradient as well as the viscous term of the momentum equation are introduced briefly in the following. The SPH approximations will be indicated by brackets $\langle>$. The properties of the centered particle is denoted by the index $i$ and the properties of the neighboring particles by the index $\mathrm{j}$. The density of a particle is directly calculated by the summation over the weights of the neighboring particles:

$$
\langle\rho\rangle_{i}=m_{i} \sum_{j} W\left(\vec{x}_{i}-\vec{x}_{j}, h\right) .
$$


This formulation conserves mass exactly and prevents a non-physical density gradient over the interface of multiphase flows, in contrast to other formulations [18].

Various approaches for the approximation of gradients, like the pressure gradient term in the momentum equation $[-\vec{\nabla} p / \rho]$ are available in literature. An approximation successfully applied to multi-phase problems was proposed by Colagrossi et al. [19]:

$$
\left\langle-\frac{\vec{\nabla} p}{\rho}\right\rangle_{i}=-\frac{1}{\rho_{i}} \sum_{j} \frac{m_{j}}{\rho_{j}}\left(p_{i}+p_{j}\right) \vec{\nabla} W\left(\vec{x}_{i}-\vec{x}_{j}, h\right) .
$$

The viscous term of the momentum equation $[\vec{\nabla} \tau / \rho$ ] contains second-order derivatives. The approximation of this term was introduced to SPH by Morris et al. [20]. It is derived from the inter particle average shear stress using a combined viscosity:

$$
\left\langle\frac{\vec{\nabla} \tau}{\rho}\right\rangle_{i}=\sum_{j} m_{j} \frac{\mu_{i}+\mu_{j}}{\rho_{i} \rho_{j}} \frac{\vec{r}_{i j} \vec{\nabla} W\left(\vec{x}_{i}-\vec{x}_{j}, h\right)}{\vec{r}_{i j}^{2}+\eta^{2}} \vec{v}_{i j} .
$$

Here $\mu$ denotes the dynamic viscosity, $\vec{r}_{i j}$ is the distance vector between the particles, $V$ denotes the particle volume, $\vec{v}_{i j}$ denotes the velocity difference and $\eta$ is a small parameter, which serves to avoid singularities.

In our SPH approach the weakly compressible SPH scheme is used, meaning that non-compressible liquids are modeled as weakly compressible. Therefore, the pressure $p$ and the density $\rho$ are linked through an equation of state. In this approach the density fluctuations are limited to $\Delta \rho / \rho=1 \%$ by imposing an artificial sound speed $c$ which is approximately ten times higher than the maximum velocity $\left|\vec{v}_{\max }\right|$. In general, this leads to an artificial sound speed, which is much lower than the physical one. By this approach small time steps due to the CourantFriedrich-Levy (CFL) criterion are mitigated.

For the present investigation the equation of state in-use is a modified Tait equation, which was originally derived for water [21]:

$$
p=\frac{\rho_{\mathrm{nom}} c^{2}}{\gamma}\left[\left(\frac{V_{\mathrm{nom}}}{V}\right)^{\gamma}-1\right] .
$$

Here $\rho_{\text {nom }}$ is the reference density, $V_{\text {nom }}$ is the reference volume and $\gamma$ is the polytropic exponent.

\section{Treatment of interfacial tension}

For the prediction of the complex physics leading to liquid atomization the modeling of surface tension effects play a crucial role. This is due to the fact, that droplet deformation and disintegration are mainly determined by the force balance between the microscopic surface force acting in tangential and normal direction at the liquid-air interface and the shear forces acting on the droplet which are induced by the air flow. This balance is described by the dimensionless Weber number $W e$, which can be used to estimate whether a droplet is exposed to an super- or subcritical aerodynamic load.

In our SPH code the surface tension is represented by the Continuum Surface Force (CSF) model, originally introduced by Brackbill et al. [22] in the framework of the VoF method. The CSF model adopted in our approach was proposed by Adami et al. [23]. The surface tension force is represented as a continuous force acting over a volume adjacent to the interface instead of a force acting directly on the surface of the droplet. Therefore, the surface tension is converted to a volumetric force $\vec{F}_{\mathrm{SF}}$ using a normalized delta-function $\delta_{\mathrm{S}}$, which has its peak at the interface:

$$
\vec{F}_{\mathrm{SF}}=\sigma \kappa \overrightarrow{\hat{n}} \delta_{\mathrm{S}} .
$$

In this formulation interfacial gradients are neglected assuming a constant surface tension coefficient. In Equation [8] $\sigma$ is the surface tension coefficient, $\overrightarrow{\hat{n}}=\vec{n} /|\vec{n}|$ is the normalized normal vector of the interface and $\kappa=-\vec{\nabla} \overrightarrow{\hat{n}}$ the curvature. The interface normal vector $\vec{n}$ is determined from the gradient of a color index $\zeta_{\mathrm{j}}^{\mathrm{i}}$, which is assigned to each SPH particle according to the phase it belongs to. This results in the following SPH approximation scheme:

$$
\vec{n}=\frac{1}{V_{i}} \sum_{j}\left(V_{i}^{2}+V_{j}^{2}\right) \frac{\rho_{i}}{\rho_{i}+\rho_{j}} \zeta_{j}^{i} \vec{\nabla} W\left(\vec{x}_{i}-\vec{x}_{j}, h\right) .
$$

The curvature $\kappa$ is determined using another approximation, described in detail by Adami et al. [23]. The additional force to be added to the momentum equation has the following form:

$$
\langle\vec{f}\rangle_{i, S F}=\frac{\vec{F}_{i, S F}}{\rho_{i}}=\frac{\sigma \kappa_{i} \overrightarrow{\hat{n}}_{i}\left|\vec{n}_{i}\right|}{\rho_{i}}=-\frac{\sigma}{\rho_{i}}\left(\vec{\nabla} \overrightarrow{\hat{n}}_{i}\right) \vec{n}_{i} .
$$

Wetting effects, which for example highly influence the primary atomization, are accounted for by using the model presented by Wieth et al. [24]. 


\section{Boundary Conditions}

The numerical representation of technical relevant systems require proper boundary conditions. All boundary conditions, which can be walls, inlets or outlets as well as periodic boundaries have to be treated specifically. The boundary conditions used for the present investigations, namely walls and permeable boundaries, will be introduced briefly in the following.

Since the SPH-method is commonly applied to unbounded or confined fluid problems, numerous treatments of wall boundaries can be found in literature. The approach in-use utilizes fixed pseudo particles placed outside of the boundary surface. These wall particles take part in the approximation to minimize to truncation error of the kernel. If a particle, representing the fluid, approaches the wall and undercuts a certain cutoff distance, a repulsive force is applied [25]. The additional force resembles a Lennard-Jones potential and acts on the center line between the fluid and wall particle under consideration.

Due to the Lagrangian nature of SPH, permeable boundary conditions cannot be handled straightforward like in Eulerian methods. Particles have to be generated at the inlet and removed at the outlet in a rate, which is equivalent to the physical flow rate. This is achieved by extending the numerical domain by so called buffer zones. The buffer zones are filled with particles, which take place in the approximation. The desired boundary conditions for the velocity $\vec{u}$, the pressure $p$ and the temperature $T$ are imposed onto these particles. The particles in the buffer zones are controlled by markers, which do not take place in the approximation and which are positioned right at the boundary surface. This procedure is suitable for arbitrarily shaped boundaries and enables the generation of particles at the inlet and the removal of particles at the outlet. A detailed description of the permeable boundaries method is given by Braun et al. [26].

\section{Modeling of the three fluid contact line}

The present investigation contains predictions of pure liquid droplets as well as of droplets containing a second phase. In the later case it is possible that three fluids, i.e. fuel, water and air, meet at one contact line. In the vicinity of this contact line, the fluids will form specific contact angles depending on the different interfacial tensions of the fluid pairings. To account for this effect, the formation of the contact angles has to be modeled. The modeling approach is presented briefly in the following.

Generally, the mechanical equilibrium state of three liquids can be expressed as a force balance of the interfacial tension forces at the contact line, where all three phases intersect. This force balance is illustrated in Figure 2.

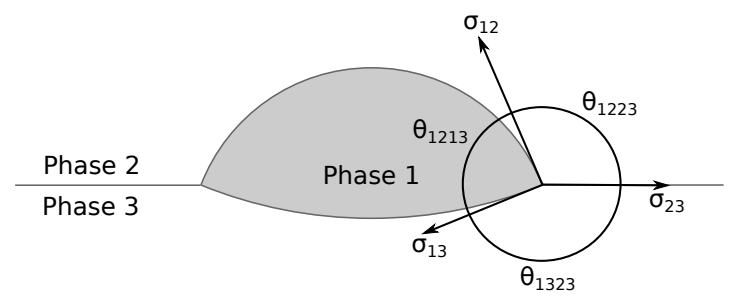

Figure 2. Schematic of the force balance at the triple line for a general three-phase interaction

In Figure 2 0 one phase (light grey, denoted as phase 1) is surrounded by two other immiscible phases (denoted as phase 2 and 3). The interfacial tension coefficients between the phases is denoted by $\sigma_{\mathrm{ij}}$, where $\mathrm{i}$ and $\mathrm{j}$ represent the index of the phases considered. The interfacial tension forces ensure the formation of an equilibrium state leading to characteristic static contact angles inside the different phases. These angles $\theta$ are indexed by the interfacial forces, which span the angles. The force balance at the triple line results in a set of three equations, which relate the interfacial tension coefficients to the static contact angles. The geometric interpretation of this set of equations is known as the Neumann triangle. For dynamic contact angle simulations the static contact angles is set as initial condition.

In our approach the surface tension is represented by the CSF model, requires an additional acceleration in the momentum equation, which primarily depends on the interface normals $\vec{n}$ and their divergence (curvature), cf. Equation 10 . In the vicinity of the triple line the normal vectors are adjusted to introduce the desired static contact angles. Up to now the modeling of fluid interactions of three liquids and/or gases has not been realized on basis of the CSF model in the SPH framework. Hu and Adams [27] showed the applicability of a different approach, the Continuum Surface Stress (CSS) model to three phase interaction problems.

A schematic representation of the normal vector correction approach for a general three phase interaction is shown in Figure 3 .

The correction of the normal vectors is only applied to particles which are close to the triple line and if particles of the other phases are located within the radius of influence, like it is the case for the black particle indicated in Figure 3. For each of those particles, two interface normal vectors $\vec{n}_{1}$ and $\vec{n}_{2}$ are calculated using (9). These span an angle $\alpha$, which does not necessarily represent the contact angle $\theta$. In order to impose a correct static contact angle, $\alpha$ has to be corrected as depicted in Figure 3 . Specifically, one of the two normal vectors, in this case $\vec{n}_{2}$, is 


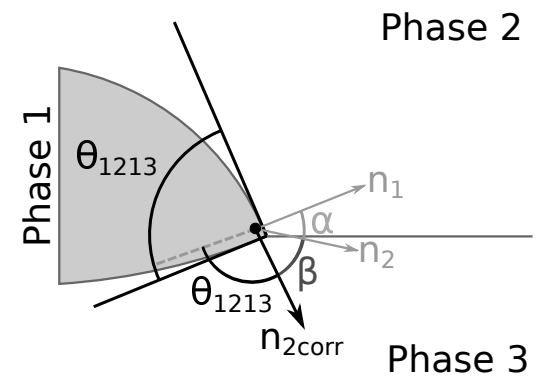

Figure 3. Illustration of the normal vector correction for the three liquid interaction case

rotated by an angle $\beta$ to the corrected normal vector $\vec{n}_{2 \text { corr }}$. The normal vector used for the rotation is chosen by the strength of the kernel support. A higher kernel support is assumed to be more trustworthy. Following the rotation of the normal vector, the general approximations are used to calculate the curvature and then the acceleration.

The model presented yields excellent results (relative errors $<5 \%$ ) in $2 \mathrm{D}$ and $3 \mathrm{D}$ for the formation of static contact angles in a water-air-alkane system. Details of the model and its validation shall be presented in a future publication.

\section{Numerical Setup}

The simulations conducted for this study are focused on the deformation behavior of single droplets in an air flow. A confined channel domain with permeable boundary conditions (inlet, outlet) is used. A sketch of the investigated geometry is depicted in Figure 4.

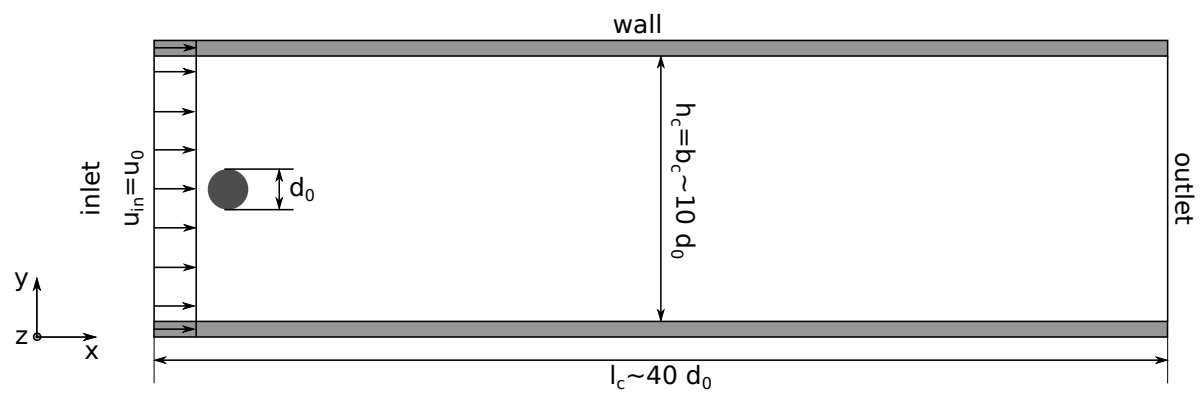

Figure 4. Illustration of the geometry and boundary conditions

The channel has a square cross-section with the height of $h_{\mathrm{c}} \approx 8.3 d_{0}=0.5 \mathrm{~mm}$ and a length of $l_{\mathrm{c}} \approx 33.3 d_{0}=2 \mathrm{~mm}$. The initial droplet diameter is denoted by $d_{0}$. The channel is confined by moving walls in y-and z-direction, which have the same velocity as prescribed at the inlet. At the inlet a fixed velocity in x-direction $u_{\text {in }}$ and at the outlet a fixed reference pressure $p_{\text {out }}$ is prescribed. The velocities imposed in this study are $u_{\text {in }}=22.5 \mathrm{~m} / \mathrm{s}$ and $u_{\text {in }}=24.34 \mathrm{~m} / \mathrm{s}$. A summary of the fluid properties can be found in Table 1. The properties of air resemble combustion chamber conditions at $T=700 \mathrm{~K}$ and $p=2 \mathrm{MPa}$.

The single droplet with a diameter of $d_{0} \approx 60 \mu \mathrm{m}$ is initialized, after the air flow has reached a quasi-steady state. Hence, the droplet is initialized by a "stamp" once the air flow is settled. Within the stamp the fluid properties are changed from air to the desired liquid. The air is put to rest for a short time. during this time span, the spurious oscillations caused by the initialization of the droplet will relax and a steady state sphere is formed. Thereafter, the air and wall particles are set to the imposed inlet velocity, so that the droplet is exposed to a sudden aerodynamic load. This method provides a fast and simple way to create different types of droplets. In this paper fuel droplets with different water volume fractions of $\phi=0, \phi=0.23$ and $\phi=1$ are investigated. This results in single fluid droplets

Table 1. Summary of fluid properties

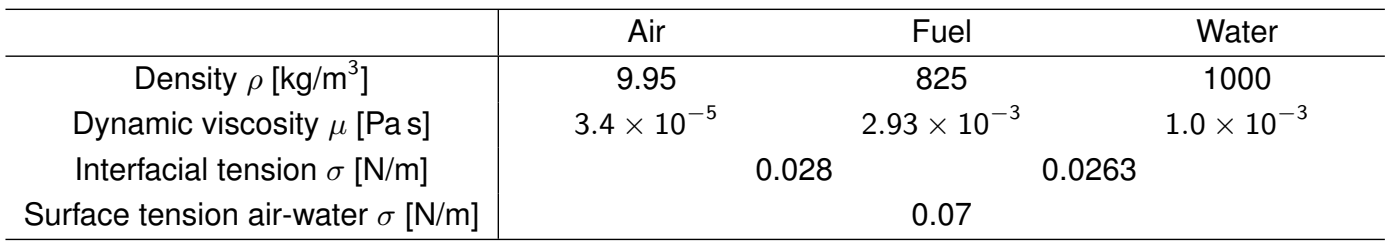


for $\phi=0$ (fuel) and $\phi=1$ (water), yielding Weber numbers for the pure fuel of $W e_{\text {fuel }} \approx 10$ and $W e_{\text {fuel }} \approx 12$ and for the water of $W e_{\text {water }} \approx 4.1$ and $W e_{\text {water }} \approx 4.8$. In the case of $\phi=0.23$ a single water droplet is added to the interior of the fuel droplet. The influence of the placement of the water is investigated for different scenarios: centered, off center downstream (in flow direction, centered in the yz-plane), off center upstream (against the flow direction, centered in the yz-plane), off center perpendicular to the flow in both y-directions (centered in the xz-plane). Overall this results in 14 simulations with each having approximately 34 million particles using an initial particle distance of $d x=2.5 \mu \mathrm{m}$. This spatial resolution is the absolute minimum required to correctly capturing the physics leading to droplet deformation or breakup. A finer spatial resolution would give even more reliable results. However, the computational effort would increase significantly. For the verification of the SPH code against common correlation other single fluid droplet simulations were conducted, which will not be specified in this context.

\section{Computational performance}

The simulations were conducted using the SPH code developed by the Institut für Thermische Strömungsmaschinen (ITS) [11]. The parallel performance of this code was evaluated by strong scalability tests performed on the ForHLR I cluster at the Steinbuch Center for Computing in Karlsruhe [28]. The cluster is equipped with 512 thin nodes, each having 2 Deca-Core Intel ${ }^{\circledR}$ Xeon $^{\circledR}$ E5-2670 v2 processors. The nodes are connected by an InfiniBand 4x FDR interconnect. For the scalability test, the SPH code was compared to the common grid based VoF solvers of OpenFOAM ${ }^{\circledR}$ 2.3.0 (interFoam) and ANSYS ${ }^{\circledR}$ Fluent $^{\circledR}$, Release 15.0.7. The domain investigated was 2D and turbulence modeling was neglected. Details of this study can be found in [29]. All simulations were run for one hour walltime, which easily enables a performance test over three orders of magnitude. Therefore, the simulation domain was divided into 1 up to 1000 subdomains. The results for the speedup and parallel efficiency are depicted in Figure 5 . The results are normalized by the performance of one node or 20 cores.

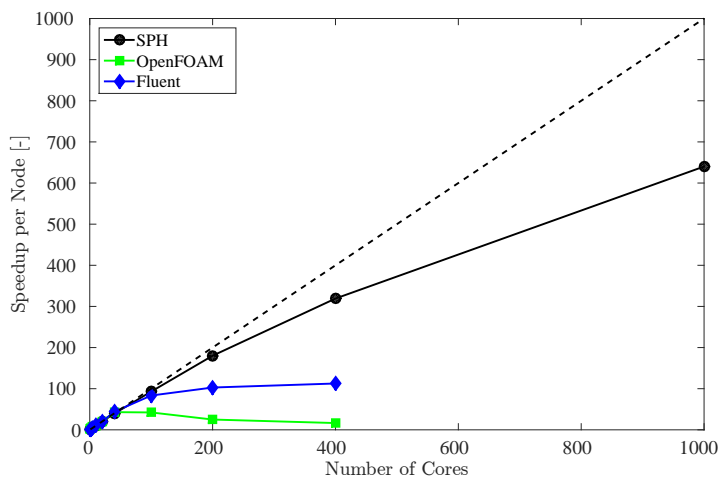

(a) Comparison of speedup per node

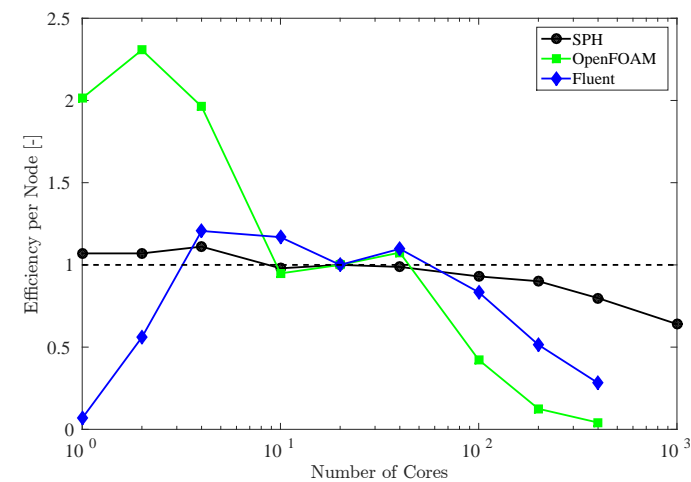

(b) Comparison of parallel efficiency per node

Figure 5. Comparison of parallel performance for SPH, OpenFOAM and Fluent. The data is normalized by the performance for one node with 20 cores

The results for OpenFOAM (indicated by squares) and Fluent (indicated by diamonds) show a good scaling for up to 2 nodes (40 processors). The speedup for SPH is almost ideal for up to 200 cores and has not jet reached saturation at 1000 cores (c.f. Figure 5(a). Consequently, the parallel efficiency of the SPH code stays above 0.9 till 200 cores, but is still over 0.6 at 1000 cores (c.f. Figure 5(b)]. The efficiency of OpenFOAM and Fluent indicates, that OpenFOAM has an excellent serial performance, while this is questionable for Fluent. Beyond 100 cores the efficiency of both codes is severely reduced and the speedup reaches saturation. This yields a stagnation (Fluent) or even increase (OpenFOAM) of time needed for the simulations when increasing the computational effort.

As it is evident, the SPH code in use shows a strong parallel performance even at high CPU numbers. Therefore, each simulation was conducted on 520 cores or 26 nodes, respectively. Depending on the simulation time, which is needed to advect the droplet through the whole domain, the average time required for the computation of each prediction is $t_{\text {Comp }} \approx 17000$ CPUh for the cases leading to $W e_{\text {fuel }}=10$ and $t_{\text {Comp }} \approx 14500$ CPUh for the cases leading to $W e_{\text {fuel }}=12$. Additionally, the computation of the quasi-stationary air solution as well as the decomposition and reconstruction of the simulation domain consumed computational resources. However, these are small compared to the resources required for the actual simulations.

\section{Results and discussion}

At first the deformation dynamics of single fluid droplets is investigated to in order to verify the SPH method, since the SPH method is relatively new to CFD. The numerical findings are compared to the well-known correlations of Hsiang and Faeth [2]. Furthermore, an enhanced characteristic deformation time is extracted from the simulations. It is found to collapse the initial deformation dynamics for all single fluid test cases to one curve. Thereafter, the 
dynamics of two fluid droplets at the same aerodynamic loading are compared to those of single fluid droplets with the objective to determine the influence of adding a second fluid.

\section{Single fluid droplet simulations}

The aerodynamic loads chosen for this investigation are characterized by Weber numbers below 12 for the pure liquids. In this We number range, droplets are expected to just show an oscillatory deformation. Breakup can only occur due to natural oscillations of the droplet [3]. The temporal evolution of the deformation as predicted by SPH for a pure fuel droplet at $W e_{\text {fuel }}=10$ is depicted in Figure 6. The air as well as wall particles are omitted for the sake of clarity.

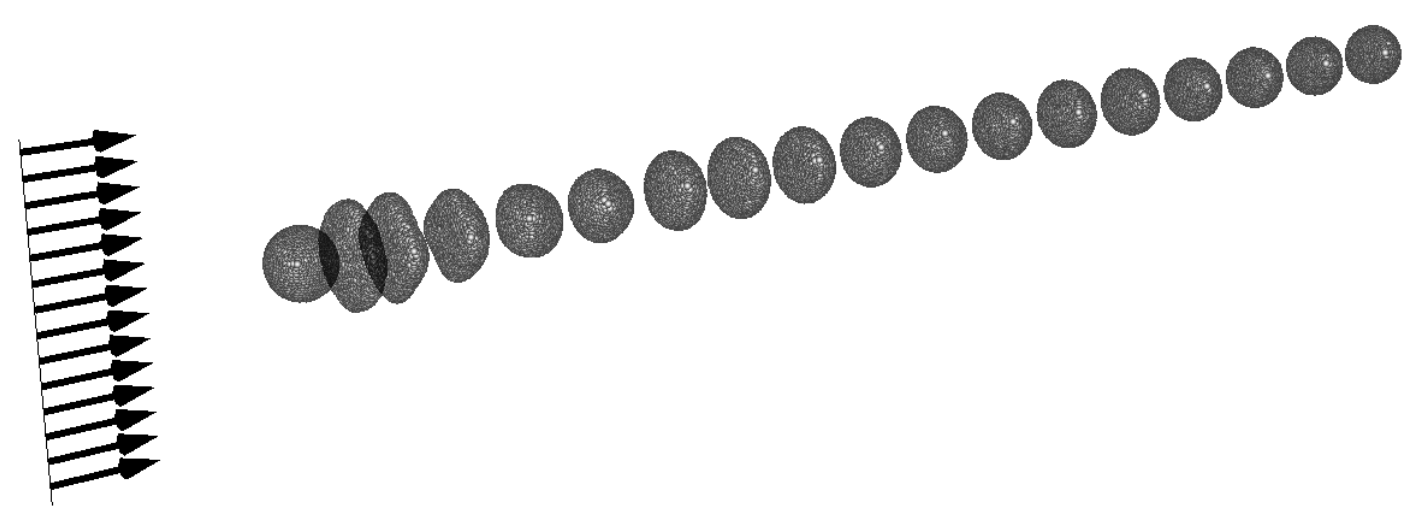

Figure 6. Pure fuel droplet evolution for $W e_{\text {fuel }}=10$

The aerodynamic load, indicated by black arrows, is impinging on the initial spherical drop in a shock-like manner. This leads to the deformation of the droplet to a flat disc. Thereafter, the surface tension causes a contraction of the droplet. The high dynamic viscosity of the fluid prevents the droplet from elongating in flow direction, yielding a spherical shape at the turning point of the deformation. Then, the drop starts to flatten again due to the forces imposed by the pressure of the air flow around the droplet and the surface tension force. Overall this leads to an oscillatory deformation, which is dampened severely by the viscosity of the fuel. A similar oscillatory deformation was also found for the other pure liquids. Thereby, the oscillation of the water droplets are of a higher frequency and dampened less as expected due to the higher surface tension and lower dynamic viscosity.

Overall the qualitatively observed deformation dynamics of the single fluid droplets perfectly reproduce the expected behavior found by experimental investigations. For the verification, that SPH is able to correctly predict the quantitative behavior of the drop deformation, the predictions are compared to empirical correlations in the following.

\section{Comparison of SPH results to empirical findings}

Hsiang and Faeth [2] determined the maximum extent of the droplet $d_{\text {cross,max }}$ perpendicular to the flow direction as well as the minimum extent $d_{\mathrm{str} \text { min }}$ in flow direction experimentally. They found that the droplet extent perpendicular to the flow almost linearly increases with time until the maximum is reached. On a basis of a phenomenological analysis considering the surface tension and pressure forces, together with the experimental results they proposed the following correlation:

$$
\frac{d_{\text {cross }, \max }}{d_{0}}=\left(\frac{d_{0}}{d_{\text {str,min }}}\right)^{0.5}=1+0.19 W e^{0.5} \text {. }
$$

The validity of this correlation is claimed to be for $W e<100$ and $O h<0.1$, where $O h$ represents the Ohnesorge number relating the viscous forces to the surface tension and inertial forces. The correlation claims, that the maximum cross-stream as well as the minimum stream-wise diameter are just dependent on $W e$. Since the four cases addressing single fluid droplets are representing just four different $W e$ and two different $O h$, more numerical predictions of single fluid droplets were conducted to cover a broader range of $W e$ as well as $O h$. The simulations cover $2<W e<12$ and $0.005 \leq O h \leq 0.1$. The comparison of the numerical results to the correlation given by [11] is depicted in Figure 7. The numerical results were obtained in a similar fashion as it is commonly done in experiments. Fixing the view to one observation plane ( $x-y$ in this case), the drop dimensions in $x$ - and $y$-direction are measured. The findings for the maximum cross-stream extent in y-direction $d_{y, \text { cross,max }}$ are shown in Figure $7(\mathrm{a})$ while the findings for $d_{\text {str,min }}$ are shown in Figure $7(\mathrm{~b})$

It is evident, that the numerical results fit the correlation extremely well. Minor deviations are observed for two points. One of them represent an extreme investigated. The droplet simulated at $W e=2$ features an Ohnesorge number $O h=0.1$, which is the limit of the correlation. This could explain the observed deviation. The second case is well in the range of validity of the correlation, having a $W e \approx 5$ and $O h \approx 0.016$. Therefore, this deviation only 


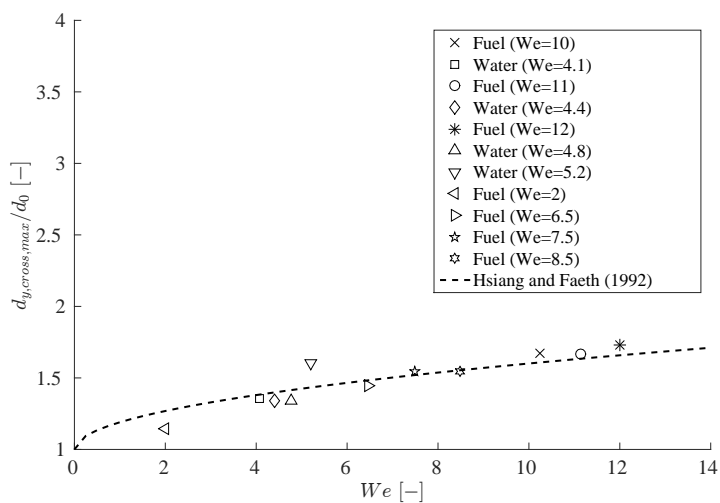

(a) Maximum droplet extent perpendicular to the flow direction $d_{\text {y,cross,max }}$ over $W e$

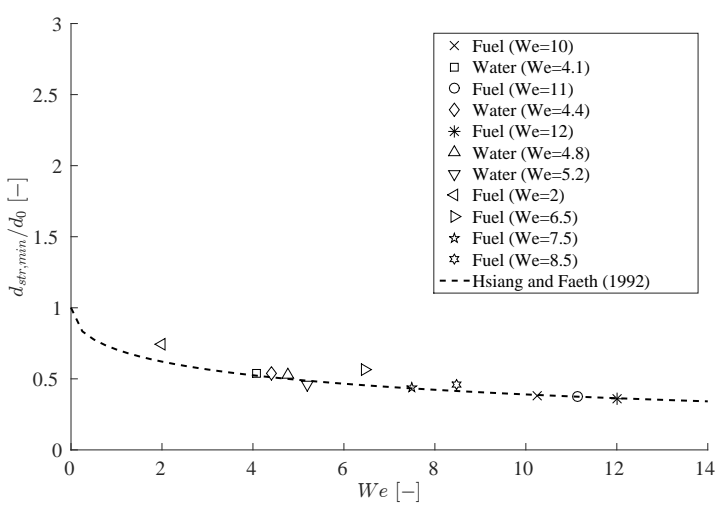

(b) Minimum droplet extent in flow direction $d_{\text {str,min }}$ over $W e$

Figure 7. Comparison of the single fluid simulation results to empirical findings of Hsiang and Faeth [2

can be explained by numerical inaccuracies resulting from different numerical setups.

Altogether, it can be stated that SPH is able to capture quantitatively the correct physical behavior of drop deformation at low aerodynamic loads. The initial temporal dynamics of the single fluid drop deformation is discussed in the following.

\section{Temporal evolution of the initial drop deformation}

The temporal evolution of the drop deformation and thus the drag coefficient plays a major role when developing simplified models, which can be used in an Euler-Lagrange context. Hsiang and Faeth [2] observed a linear increase in cross-stream diameter with time until the maximum deformation is reached. They claim, that for a wide range of $W e$ the maximum deformation is always reached at approximately $t / t * \approx 1.6$, where $t *=d_{0}\left(\rho_{\text {liq }} / \rho_{\text {gas }}\right)^{0.5} / u_{0}$ is the characteristic breakup time proposed by Ranger and Nicholls [30]. The temporal evolution of the deformation perpendicular to the flow in $y$-direction $d_{y, \text { cross }}$ for all single fluid cases is depicted in Figure 8

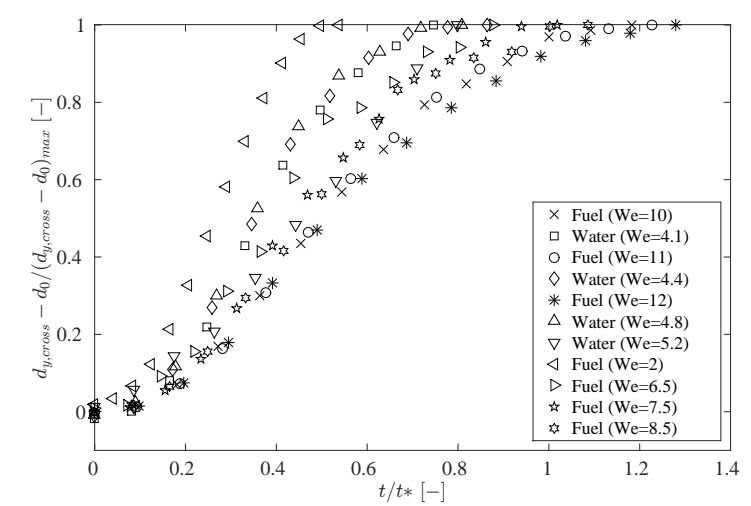

(a) Time evolution of the initial droplet deformation perpendicular to the flow in y-direction

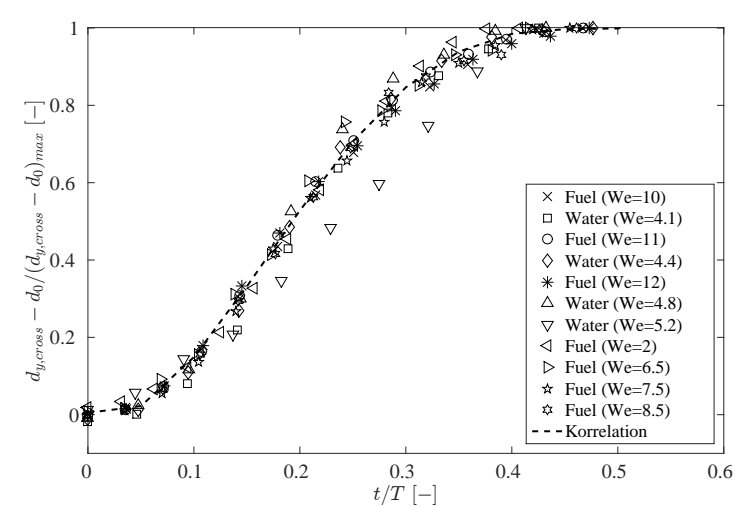

(b) Correlated time evolution of the initial droplet deformation perpendicular to the flow in y-direction

Figure 8. Temporal evolution of the droplet deformation perpendicular to the flow direction

The numerical results for the cross-stream deformation over the dimensionless time $t / t *$ are shown in Figure $8(\mathrm{a})$. As evident, the dynamics of the deformation predicted by SPH is not linear and cannot be correlated using the commonly used characteristic time $t *$. Furthermore, overall the deformation as predicted seems to be faster than the experimental findings, exhibiting $t / t * \approx 1.6$ for the maximum deformation [2]. The maximum dimensionless time needed for the maximum deformation in $\mathrm{SPH}$ is $t / t * \approx 1.3$. The deviations observed may be due to the experimental setup used to acquire the data. Commonly shock tube experiments are used for the aerodynamic loading of droplets. These kind of experiments cannot guarantee correct boundary conditions in contrast to the numerical analysis. Furthermore, the droplets are introduced into the shock tube by a droplet chain using a vibrating capillary tube. This might lead to an interference due to previous droplets or the vibration might influence the initial drop shape.

A closer look to the dynamics in Figure $8(\mathrm{a})$ indicates a dependence on $W e$ for the initial deformation dynamics. 
The smaller $W e$ the faster the maximum cross-stream deformation is reached. Correlating the numerical results with $t *$ and $W e$ using a 4th order polynomial leads to the following enhanced characteristic time:

$$
T=W e^{0.4} d_{0}\left(\rho_{\text {liq }} / \rho_{\text {gas }}\right)^{0.5} / u_{0}=W e^{0.4} t * .
$$

The plot of the predicted deformation dynamics over the new dimensionless time $t / T$ are depicted in Figure 8(b). Now the deformation dynamics of all cases coincide on one curve. The only one case showing a deviation is the same as before $(W e \approx 5 ; O h \approx 0.016)$. Here, too the probable cause is numerical inaccuracies.

The new characteristic time was derived using the data for the cross stream deformation of the droplets. In Figure 9 the temporal evolution of the droplet dynamics in flow direction is plotted over the dimensionless time. In Figure $9(\mathrm{a})$ $t / t *$ is used as dimensionless time while in Figure $9(\mathrm{~b}) t / T$ is used.

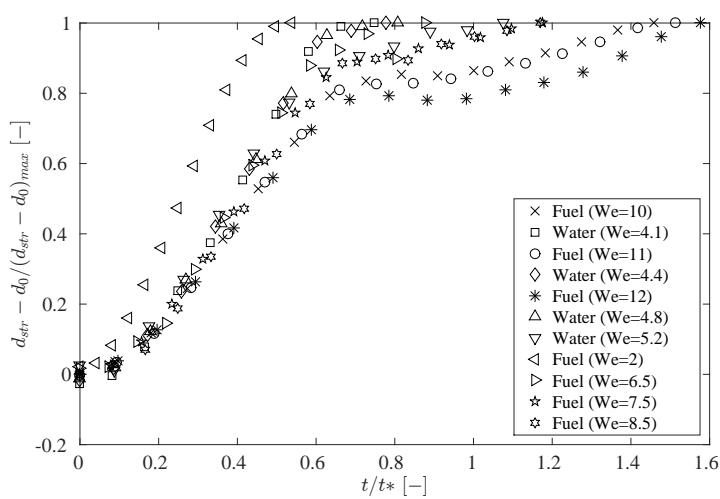

(a) Time evolution of the initial droplet deformation in flow direction

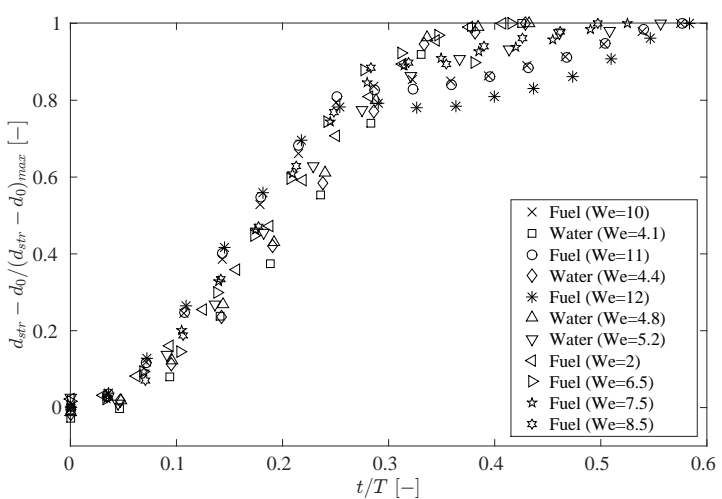

(b) Correlated time evolution of the droplet deformation dynamics in flow direction

Figure 9. Temporal evolution of the droplet deformation in flow direction

Apparently, here too the new characteristic time serves to describe the dynamics of the deformation better, collapsing the temporal evolution to one curve. Even the formation of a small bag in upstream direction, which is observed in most cases, is reproduced quite well. The bag formation is indicated by the decrease of deformation at about $t / T \approx 0.3$ in Figure $9(\mathrm{~b})$. The bag is formed by a faster acceleration of the outer part of the droplet compared to the core part. Although the small bag is formed, a droplet flattening is observed. Due to the ongoing droplet deformation perpendicular to the flow direction, the curvature on the upstream side of the droplet is decreased. Such a bag formation was not observed experimentally, whereas this phenomenon occurred in other numerical investigations as well [6, 4].

In summary it can be stated, that the dynamics of the initial deformation of single fluid droplets is dependent on $W e$ and is nonlinear with time. By introducing the new non dimensional time $t / T$ the different cases considered can be correlated quite well.

\section{Two fluid droplet simulations}

The influence of adding water to the droplet is investigated for the two different aerodynamic loads and five different placements of a single water droplet inside the fuel droplet. Exemplary the SPH prediction of temporal evolution of a fuel droplet with water added at the center at $u_{0}=22.5 \mathrm{~m} / \mathrm{s}\left(W e_{\text {fuel }}=10\right)$ is depicted in Figure 10

The addition of water causes a difference in deformation behavior. Due to the higher density as well as viscosity the dispersed water is deformed slower. This leads almost to a separation of the two phases (c.f. second instance in Figure 10, which is counteracted by interfacial forces acting on the different fluid pairings. In the further course of the simulation fuel and water are unified again and the droplet shows oscillatory deformations, like it was observed for the pure liquid cases. The deformation behavior for all the other two fluid droplet simulations is basically similar with minor differences due to the water placement. The water droplets placed off center perpendicular to the flow direction, additionally feature a rotation of the whole droplet. Due to the spatial resolution, which is not sufficient to properly resolve the three-liquid contact line, the behavior of these droplets might not be physical. Therefore, these results will be left out in the following.

In Figure 11 the deformation of the droplets in flow direction $d_{\mathrm{str}}$ and perpendicular to the flow $d_{\mathrm{y}, \text { cross }}$ over time for an air velocity of $u_{0}=22.5 \mathrm{~m} / \mathrm{s}$ is depicted. In Figure 11(a) the deformation in flow direction while in Figure 11(b) the deformation perpendicular to the flow in y-direction is plotted.

The pure liquid cases are indicated by black crosses (fuel) and blue squares (water). It is evident, that the water oscillates at a higher frequency and the oscillation is less dampened due to the lower $O h$. In case of the two fluid droplets, a the damping of the oscillation is similar to that of the pure fuel droplets. The frequency of the deformation seems to be in-between the frequencies of water and fuel for the first two oscillations. Afterwards, the oscillations 


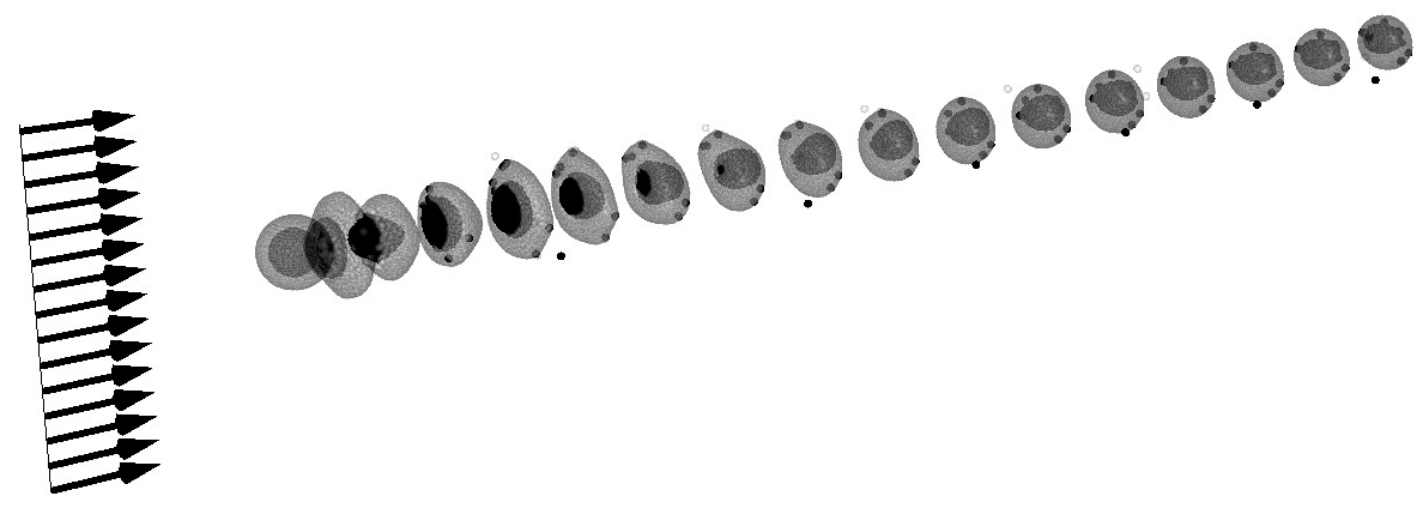

Figure 10. Temporal droplet evolution of a fuel droplet with water centered at $u_{0}=22.5 \mathrm{~m} / \mathrm{s}$

almost vanish or show a behavior which cannot be classified by the behavior of neither fuel nor water. Possibly, the behavior is triggered by single water particles located at the surface of the fuel. These single particles do not represent a physical water droplet. They are rather the result of the insufficient spatial resolution of the three fluid contact line. Therefore, a non-physical acceleration due to the CSF model is imposed (c.f. Figure 10).

The deviations observed for the initial droplet extent perpendicular to the flow and in flow direction in Figure 11 are due to the droplet initialization described previously. During the formation of the steady state droplet, some particles of the water droplet placed eccentric in the fuel droplet have air particles within their radius of influence. Therefore, due to the surface tension modeling an ellipsoid rather than a spherical droplet is formed.

The dynamics of the initial deformation shows an ambivalent behavior. In flow direction the deformation dynamics as well as the minimum extent of the two fluid droplets are similar to the results of the water. Perpendicular to the flow the droplet deformation and maximum droplet extent are similar to the observations made for the fuel droplet. At a first glance, this indicates similar drag coefficients for the two fluid and fuel droplets in the initial deformation stage. Therefore, a description of two fluid droplets might be possible by currently used simplified models.

Whether the same observations are true for the whole range of low $W e \leq 12$, is revealed by the results with higher aerodynamic loading investigated in this study. In Figure 12 the droplet deformation in flow direction $d_{\text {str }}$ and perpendicular to the flow $d_{y \text {,cross }}$ over time is plotted for an air velocity of $u_{0}=24.34 \mathrm{~m} / \mathrm{s}$. Here too, the deformation dynamics in flow direction is shown in Figure 12(a) and the deformation in cross-stream direction in Figure 12(b). The deformation behavior of the pure liquids (water: blue squares and fuel black crosses) show a similar behavior as for the lower aerodynamic load. Similarly, the water droplet exhibits a distinct higher oscillation frequency and lower damping of the oscillation than the fuel droplet. Only the initial amplitude of the deformation is increased, as evident from correlation 12.

Regarding the deformation of the two fluid droplets, leaving out the cases with water placed off center perpendicular to the flow again, the initial deviations of the droplet extents as well as the dynamics observed are similar to the cases with the lower aerodynamic load. Here too, the differences of the initial extents is also a result of the initialization of

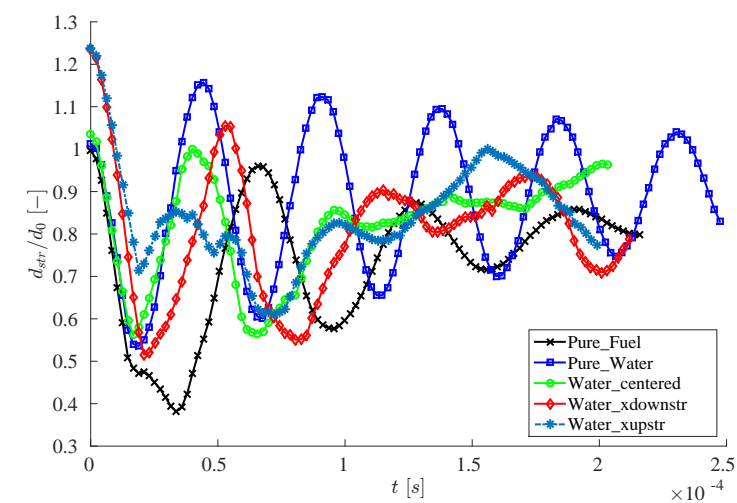

(a) Temporal evolution of the droplet deformation in flow direction $d_{\text {str }}$

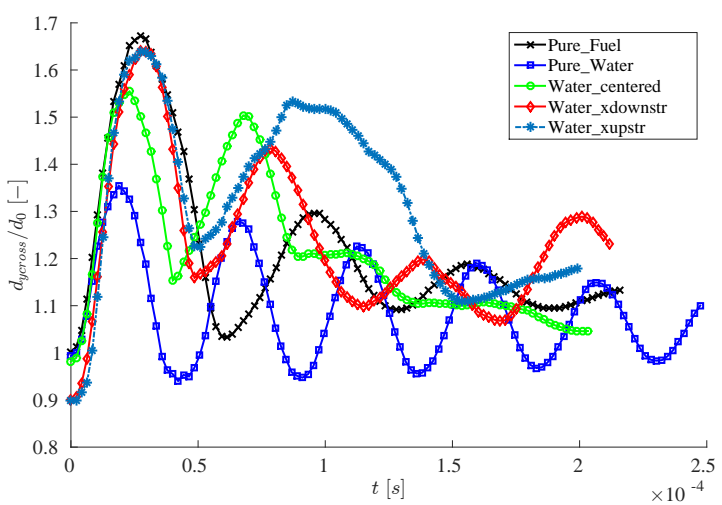

(b) Temporal evolution of the droplet deformation perpendicular to the flow in y-direction $d_{y, \text { cross }}$

Figure 11. Droplet deformation over time for all cases investigated at $u_{0}=22.5 \mathrm{~m} / \mathrm{s}$ 


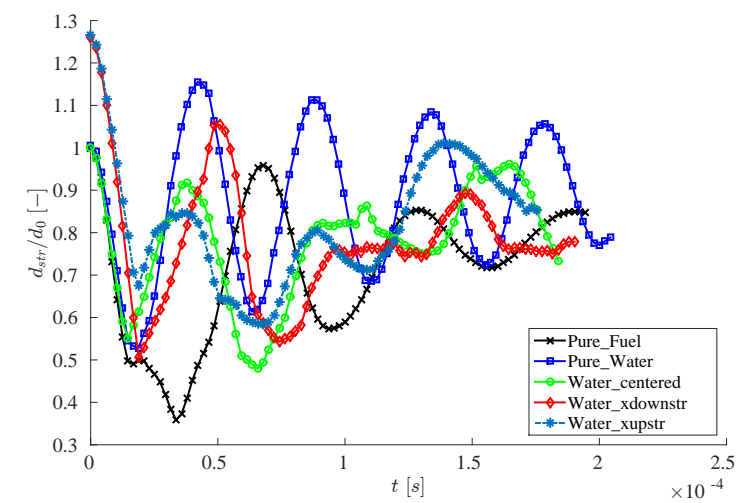

(a) Temporal evolution of the droplet deformation in flow direction $d_{\mathrm{str}}$

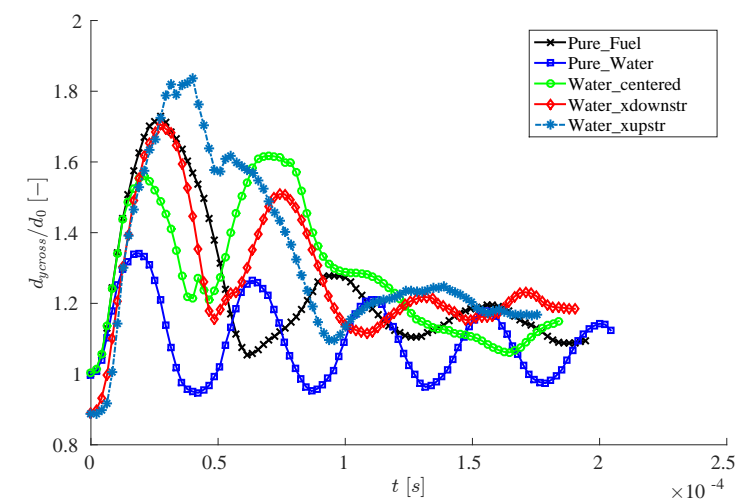

(b) Temporal evolution of the droplet deformation perpendicular to the flow in y-direction $d_{y, \text { cross }}$

Figure 12. Droplet deformation over time for all cases investigated at $u_{0}=24.34 \mathrm{~m} / \mathrm{s}$

the droplet at the start of the simulation. The frequency of the first two oscillations is again somewhere in between the dynamics of pure fuel and pure water. Furthermore, the damping of the amplitude is as strong as in the case of the pure fuel, resembling the behavior observed before. Furthermore, the amplitude of the deformation exhibits the same ambivalent behavior as in the previous cases. The minimum extent in flow direction is similar to that of the pure water case, whereas the maximum cross stream extent is similar to the deformation of the pure fuel droplet. In all cases investigated $\left(u_{0}=22.5 \mathrm{~m} / \mathrm{s}\right.$ and $\left.u_{0}=24.34 \mathrm{~m} / \mathrm{s}\right)$ the droplets just experience deformation and no droplet breakup is observed. Since by SPH a droplet breakup is predicted at $W e=13$ it is assumed that the addition of $23 \%$ volume fraction of water does not change the characteristics of the droplet dynamics described by the pure fuel Weber number $W e_{\text {fuel }}$, at least in the deformation regime. Furthermore, it may be concluded from the deformation dynamics of the two fluid droplets, that they experience a similar drag coefficient as pure fuel droplets in the early stages of the deformation. This fact would allow to use the common correlations with minor adaptations in Euler-Lagrange investigations for two fluid droplets as well.

\section{Conclusion}

In this paper the dynamics of one and two fluid droplets at low aerodynamic loads is investigated numerically using the Smoothed Particle Hydrodynamics (SPH) method. The presented predictions are focused on the deformation dynamics of pure liquid and water-in-fuel droplets with a diameter of $d_{0} \approx 60 \mu \mathrm{m}$ exposed to two different air flow velocities: $u_{0}=22.5 \mathrm{~m} / \mathrm{s}$ and $u_{0}=24.34 \mathrm{~m} / \mathrm{s}$. As the SPH method is relatively new to CFD applications, first a verification of the code in-use is done comparing numerical results for pure liquid droplets to well known empirical findings. With few exceptions the predicted minimum initial deformation in flow direction $d_{\text {str }}$ as well as the maximum cross-stream deformation $d_{\text {cross }}$ perfectly matches the correlation of Hsiang and Faeth [2]. Deviations are observed mainly to occur at extreme conditions. The results demonstrate the capability of SPH for capturing the droplet deformation dynamics. Second, the dynamic deformation of the single fluid droplets was analyzed and found to be dependent on $W e$. A new correlation for the droplet deformation was proposed. This correlation uses a modified definition of the characteristic time $T$ compared to the correlations of Hsiang and Faeth [2].

For the prediction of two fluid droplets, a single water droplet with a volume fraction of $23 \%$ was added to a fuel droplet and the placement was varied. For both aerodynamic loads the two fluid droplets show a behavior, which can be classified by the pure fuel Weber number $W e_{\text {fuel }}$. The dynamic behavior of the two fluid droplets qualitatively feature a frequency in between the fuel and water droplet for the first oscillations, while the damping of the oscillations is similar to the fuel droplet. Furthermore, an ambivalent behavior is observed for the minimum extent in flow direction and the maximum cross-stream deformation. In the first case the two fluid droplets behave as the water droplet, while in the later case their behavior is similar to the fuel droplet.

In general, it can be stated that the SPH code is capable of predicting multiphase flows, like the droplet deformation, physically correct. Therefore, SPH will be used as tool to further investigate the dynamics of droplet deformation of mono- and two fluid droplets in order to derive simpler models, which can be used in typical CFD predictions of sprays.

\section{Acknowledgement}

The financial support of the German Federal Ministry of Economics and Technology and Siemens AG within the cooperative research project 'Entwicklung von Verbrennungstechnologien im CEC für klimaschonende Energieerzeugung (03ET7011E)' is gratefully acknowledged. 
This work was performed on the computational resource ForHLR Phase I, funded by the Ministry of Science, Research and the Arts Baden-Württemberg and DFG ("Deutsche Forschungsgemeinschaft").

\section{Nomenclature}

$c \quad$ artificial sound speed $\left[\mathrm{m} \mathrm{s}^{-1}\right]$

$d x \quad$ mean particle spacing [m]

$f \quad$ spatial function [-]

$d \quad$ diameter [m]

$h \quad$ smoothing length [m]

$l \quad$ length [m]

$\vec{n} \quad$ normal vector [m]

Oh Ohnesorge number [-]

$p$ pressure $[\mathrm{Pa}]$

$t \quad$ time $[\mathrm{s}]$

$\vec{u} \quad$ velocity $\left[\mathrm{ms}^{-1}\right]$

$V \quad$ volume $\left[\mathrm{m}^{3}\right]$

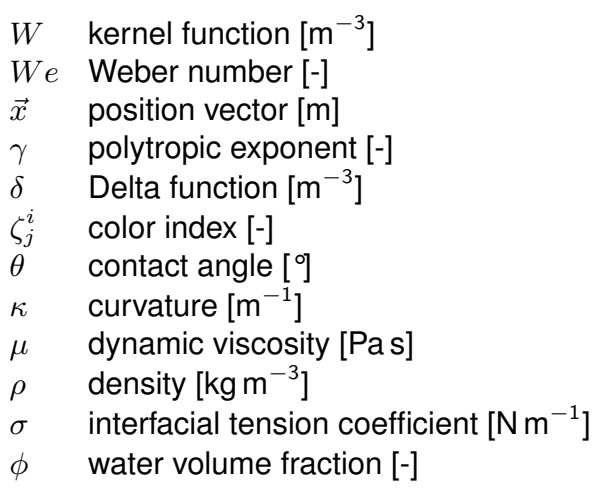

\section{References}

[1] Hinze, J. O., 1955, AIChE Jounal, 1, pp. 289-295.

[2] Hsiang, L.-P. and Faeth, G. M., 1992, International Journal of Multiphase Flow, 18(5), pp. 635-652.

[3] Guildenbecher, D. R., López-Rivera, C. and Sojka, P. E., 2009, Experiments in Fluids, 46, pp. 371-402.

[4] Zaleski, S., Li, J. and Succi, S., 1995, Physical Review Letters, 75(2), pp. 244-247.

[5] Quan, S. and Schmidt, D.P., 2006, Physics of Fluids, 18, 102103.

[6] Khare, P., Ma, D., Chen, X. and Yang, D., Sep. 2.-6. 2012, 12th Triennial International Conference on Liquid Atomization and Spray Systems.

[7] Schmehl, R., Maier, G. and Wittig, S., July 2000, 8th International Conference on Liquid Atomization and Spray Systems.

[8] Schmehl, R., Sep. 9.-11. 2002, 18th Annual Conference on Liquid Atomization and Spray Systems.

[9] O'Rourke, P.J. and Amsden, A.A., November 1987, International Fuels and Lubricants Meeting and Exposition.

[10] Bartz, F.-O., Schmehl, R., Koch, R. and Bauer, H.-J., September 2010, 23rd Annual Conference on Liquid Atomization and Spray Systems.

[11] Höfler, C., Braun, S., Koch, R. and Bauer, H.-J., 2013, Journal of Engineering for Gas Turbines and Power, 135.

[12] Lechner, C. and Seume, J., 2010, "Stationäre Gasturbinen". Springer Verlag

[13] Dryer, F. L., 1977, Symposium (International) on Combustion, 16(1), pp. 279-295.

[14] Gingold, R.A. and Monaghan, J. J., 1977, Monthly Notices of the Royal Astronomical Society, 181, pp. 375-389.

[15] Lucy, L.B., 1977, The Astronomical Journal, 82, pp. 1013-1024.

[16] Liu, M.B. and Liu, G.R., 2010, Archives of Computational Methods in Engineering, 17, pp. 25-76.

[17] Monaghan, J.J., 1992, Annual Review of Astronomy and Astrophysics, 30, pp. 543-574.

[18] Hu, X.Y. and Adams, N.A., 2007, Journal of Computational Physics, 227, pp. 264-278.

[19] Colagrossi, A. and Landrini, M., 2003, Journal of Computational Physics, 191, pp. 448-475.

[20] Morris, J.P., Fox, P.J. and Zhu, Y., 1997, Journal of Computational Physics, 136, pp. 214-226.

[21] Batchelor, G. K., 2000, "An Introduction to Fluid Dynamics". Cambridge University Press

[22] Brackbill, J.U., Kothe, D.B. and Zemach, C., 1992, Journal of Computational Physics, 100, pp. 335-354.

[23] Adami, S., Hu, X.Y. and Adams, N.A., 2010, Journal of Computational Physics, 229, pp. 5011-5021.

[24] Wieth, L., Braun, S., Koch, R. and Bauer, H.-J., September 2014, 26th European Conference on Liquid Atomization and Spray Systems.

[25] Monaghan, J. J., 1994, Journal of Computational Physics, 110, pp. 399-406.

[26] Braun, S., Wieth, L., Koch, R. and Bauer, H.-J., June 2015, 10th International SPHERIC Workshop.

[27] Hu, X.Y. and Adams, N. A., 2006, Physics of Fluids, 18, pp. 101702.

[28] Forschungshochleistungsrechner ForHLR Phase I http://www.bwhpc-c5.de/wiki/index.php/ForHLR Phase_I_-_Hardware_and_Architecture ([cit. 2016-04-04]).

[29] Braun, S., Krug, M., Wieth, L., Höfler, C., Koch, R. and Bauer, H.-J., August 2015, 13th Triennial International Conference on Liquid Atomization and Spray Systems.

[30] Ranger, A.A. and Nicholls, J.A., 1969, AIAA Jounal, 7(2), pp. 285-289. 